\title{
Serum Levels of Syndecan-1, Interleukin-6 and C-Reactive Protein in Statin-Treated Patients with Type 2 Diabetes Mellitus
}

\author{
Mohamed AbdEllatif Mahmoud* \\ Medical Research Institute, Alexandria University, Egypt
}

Received: December 09, 2015; Accepted: December 15, 2015; Published: December 19, 2015

*Corresponding author: Mohamed AbdEllatif Mahmoud, Medical Research Institute, Alexandria University, Egypt

E-mail: mohamed_hadad1980@yahoo.com

This study was undertaken to evaluate the alterations of serum levels of syndecan-1, lipids, interleukin- 6 and C-reactive protein in type 2 diabetic patients and to assess the possible effect of statins on syndecan-1 level targeting the prevention of pathologic inflammatory events in these patients.

The present study conducted on 40 patients with type 2 diabetes mellitus and 10 healthy controls. The patients group was subdivided into 2 groups; the first included 20 type 2 diabetic patients treated with conventional therapy of diabetes, while, the other comprised 20 type 2 diabetic patients treated with conventional therapy of diabetes together with simvastatin with a dose of $40 \mathrm{mg}$ per day for 10 weeks.

To all studied subjects, detailed history and full clinical examination were done. Some laboratory investigations were also done at the beginning of the study and repeated after 10 weeks of treatment for both groups of patients including: serum levels of glucose fasting and post prandial, Glycated Hemoglobin (HbA1c), Total Cholesterol (TC), high and low density lipoprotein cholesterol (HDL-C and LDL-C), Triglycerides (TG), Apolipoprotein-A1 (apo-A1) and Apolipoprotein-E (apo-E). In addition, urinary albumin to Creatinine Ratio (ACR) was estimated. Serum levels of inflammatory biomarkers were also estimated included high sensitivity C-Reactive Protein (hs-CRP) and Interleukin-6 (IL-6). Moreover, serum level of syndecan-1 was also measured by using Enzyme-Linked Immunosorbent Assay (ELISA).

The present study revealed significant increase in FBG, PPG and $\mathrm{HbA} 1 \mathrm{c}$ in both groups of diabetic patients at base line values compared to controls. Significant reduction in serum levels of TC, LDL-C, TG and apo-E and significant elevation in HDL-C and apo-A1 were observed in diabetic patients receiving statin in combination with the conventional treatment of diabetes compared to the corresponding values before starting statin treatment. In contrast, there were no significant changes in these parameters after 10 weeks in diabetic patients receiving conventional treatment of diabetes alone compared to the corresponding base line values.

Regarding inflammatory biomarkers, serum levels of hsCRP and IL-6 demonstrated significant elevation in both groups of diabetic patients at the beginning of the study compared to controls, while, they showed significant reduction in diabetic patients receiving statin in combination with the conventional treatment of diabetes compared to the corresponding values before starting statin treatment. After 10 weeks of starting the study, no significant changes in inflammatory markers were observed in patients receiving conventional treatment of diabetes solely compared to the corresponding values at the beginning of the study.

Serum levels of soluble syndecan-1 showed significant reduction in both groups of diabetic patients at base line values compared to controls, while, syndecan-1 level showed significant elevation in diabetic patients receiving statin in combination with the conventional treatment of diabetes compared to the corresponding mean value before starting simvastatin treatment. After 10 weeks of starting the study, no significant change in syndecan-1 was observed in patients receiving conventional treatment of diabetes solely compared to the corresponding mean value at the beginning of the study.

\section{Conclusion}

From the results obtained in the present study, it could be concluded that statin-mediated increase of syndecan-1 shedding could serve as a new target for the prevention of atherosclerosis and associated-pathologic inflammatory events in type 2 diabetes. Although the present study could throw the light upon the importance of syndecan- 1 as anti-atherosclerotic and antiinflammatory modulator in type 2 diabetes mellitus, the exact nature, magnitude and role of syndecan- 1 need further intense investigations. 